\title{
Serum and bone pentosidine in patients with low impact hip fractures and in patients with advanced osteoarthritis
}

\author{
Jan Vaculík ${ }^{1}$, Martin Braun ${ }^{2}$, Pavel Dungl ${ }^{1}$, Karel Pavelka ${ }^{3}$ and Jan J. Stepan ${ }^{3^{*}}$
}

\begin{abstract}
Background: Femoral neck fractures are a common occurrence in patients suffering from osteoporosis, while intracapsular hip fracture is rare in cases of osteoarthritis of the hip. Previous histomorphometric studies have emphasized the association between bone microarchitecture and the risk of low-impact fractures in osteoarthritis and osteoporosis patients. However, the strength of bone material is also a function of composition of organic bone matrix. In order to compare tissue material properties in these two clinical conditions, serum and bone pentosidine, a non-enzymatic collagen crosslinking element, was measured in patients who suffered a low-impact fracture, and in patients with advanced osteoarthritis.
\end{abstract}

Methods: The patient population consisted of 70 patients who underwent hemiarthroplasty surgery for a femoral neck fracture, and 41 patients with advanced hip joint osteoarthritis without a history of low- impact fracture, who were indicated for total hip joint replacement. Pentosidine content was analyzed in bone samples and in serum obtained from fracture and osteoarthritis patients using high performance liquid chromatography.

Results: Serum and bone concentrations of pentosidine were higher in subjects with hip fractures compared with osteoarthritis after adjustment for age, sex, weight, serum creatinine, and diabetes. A significant positive correlation was found between bone and serum pentosidine in fractured cases. A comparable relationship was also demonstrated for pentosidine levels in serum and bone relative to differentiation of fracture and osteoarthritis cases.

Conclusions: Serum pentosidine can be considered a potential biomarker for identification of subjects with impaired bone quality and bone strength.

Keywords: Biomarkers, Osteoarthritis, Osteoporosis, Pentosidine, Proximal femoral fracture

\section{Background}

The strength of bone material is a function of bone microarchitecture, and tissue material properties. At the material level, bone strength is determined by the degree of mineralization of basic structural units, microdamage accumulation, and properties of the organic bone matrix, namely collagen cross-link formation $[1,2]$. These are regulated by cellular activities and tissue turnover rate [3]. Impaired enzymatic cross-linking and/or increases in non-enzymatic cross-links in bone collagen

\footnotetext{
* Correspondence: stepan@revma.cz

${ }^{3}$ Institute of Rheumatology, Prague, and Faculty of Medicine 1, Charles

University Prague, Na Slupi 4, Prague CZ 12850, Czech Republic

Full list of author information is available at the end of the article
}

have been proposed as a determinant of impaired bone mechanical properties in aging, involutional osteoporosis, and diabetes mellitus $[4,5]$. Reduced bone turnover allows the formation of additional collagen crosslinks by non-enzymatic means, resulting in accumulation of nonenzymatic advanced glycation end-products (AGEs) in bone tissue [6,7]. Bone collagen glycation allows microdamage in bone to spread more easily, therefore increasing the total crack surface density, making bone tissue more brittle and more likely to fracture $[8,9]$.

Out of several AGE crosslinks, pentosidine (PEN) has been quantified in bone [10], and its accumulation has been associated with the age-related degradation of bone mechanical properties $[11,12]$. PEN is a senescent 
non-enzymatic cross-link resulting from a spontaneous interaction between arginine and lysine amino acids and free sugars [13, 14]. PEN has been shown to accumulate with age in cortical bone of human femurs [15]. Accumulation of AGEs in bone collagen matrix has been associated with brittleness of collagen fibers and impairment of the mechanical properties of cortical and trabecular bone $[11,12,16,17]$. Increased content of PEN in cortical and trabecular bone was associated with impairment of bone quality in osteoporotic patients with hip fractures [18], while plasma PEN levels were not increased in patients with osteoarthritis compared with controls [19]. However, serum and bone PEN has not been compared previously in patients with hip osteoarthritis and low-impact hip fractures.

To examine the performance of PEN as a biomarker of impaired quality of organic bone matrix, potentially associated with fracture risks, we compared PEN levels in serum and proximal femoral bone specimens using high performance liquid chromatography (HPLC) in subjects who had undergone hemiarthroplasty for an intracapsular hip fracture and from a group of fracturefree patients with hip joint osteoarthritis.

\section{Methods}

\section{Study participants}

This cohort study was performed in patients followed as part of routine care in the Department of Orthopedics, Bulovka Hospital, Prague, Czech Republic. Serum and femoral head bone specimens were collected from 70 subjects who had undergone hemiarthroplasty for an intracapsular hip fracture. Simultaneously, bone specimens from the iliac crest were obtained in random from 21 patients. The group with hip joint osteoarthritis, which was without a history of low energy fractures, included 41 patients in advanced stages of hip osteoarthritis, who were indicated for total hip joint replacement, $63 \%$ of the atrophic type, $24 \%$ of the hypertrophic type [20]. A complete clinical history, including details of specific common medical conditions (e.g., hypertension, congestive heart failure, heart attack, and stroke, diabetes mellitus), lifestyle risk factors including smoking (current, past, never) and alcohol consumption, detailed personal history of glucocorticoids use (previous or ongoing), fracture history (type and trauma), and physical examination were assessed. Excluded were patients with Paget's disease of the bone, malignancy, renal osteodystrophy, hyperparathyroidism, active liver disease or clinical jaundice, previous treatment with aminobisphosphonates, denosumab, teriparatide, vitamin $\mathrm{D}>50,000 \mathrm{IU} /$ week or active vitamin $\mathrm{D}$ analogs. Characteristics of the study subjects are shown in Table 1.

\section{Preparation of samples}

Bone specimens obtained from the femoral head of femoral neck fracture patients and specimens from the femoral head of patients undergoing total hip replacement for hip joint osteoarthritis were obtained from the central part of the femoral head using a trephine, along the femoral neck axis. The samples were taken more than $5 \mathrm{~mm}$ away from the osteotomy plane of the total hip arthroplasty to avoid heat damage to the bone specimen. In addition to bone from femoral heads, full thickness bone specimens from the anterior ilium were harvested $2 \mathrm{~cm}$ posterior and $2 \mathrm{~cm}$ inferior to the anterior superior iliac spine from 21 patients with hip fractures, using a Rochester Bone Biopsy Trephine. The bone was cleaned of adhering soft tissues and marrow. Bone specimens

Table 1 Characteristics of the study population

\begin{tabular}{|c|c|c|c|c|}
\hline & \multicolumn{2}{|l|}{ Male } & \multicolumn{2}{|l|}{ Female } \\
\hline & Fracture & Controls Osteoarthritis & Fracture & Controls Osteoarthritis \\
\hline Number of subjects & 21 & 25 & 49 & 15 \\
\hline Age (years) & $77.3 \pm 9,1^{a}$ & $64.3 \pm 10.9$ & $77.3 \pm 11.0$ & $68.0 \pm 7.9$ \\
\hline Height (cm) & $174.0 \pm 7.0$ & $176.0 \pm 5.1$ & $161.9 \pm 6.9$ & $162.9 \pm 7.5$ \\
\hline Weight (kg) & $75.9 \pm 15.0^{b}$ & $89.7 \pm 11.5$ & $68.7 \pm 15.9$ & $79.0 \pm 18.0$ \\
\hline Prevalent fracture (No.) & 2 & 0 & $21^{a}$ & 0 \\
\hline Glucocorticoids (No.) & 1 & 0 & 6 & 1 \\
\hline Smoking (No.) & 2 & 2 & 5 & 1 \\
\hline Diabetes (No.) & 3 & 6 & 11 & 3 \\
\hline Creatinine $(\mu \mathrm{mol} / \mathrm{l})$ & $107 \pm 33.9$ & $97.6 \pm 21.7$ & $90.9 \pm 29.1$ & $79.6 \pm 9.1$ \\
\hline \multirow[t]{2}{*}{ Bone pentosidine (nmol/g dry bone mass) ${ }^{c}$} & $2.96^{a}$ & 1.10 & $2.57^{a}$ & 0.93 \\
\hline & $0.55 ; 6.80$ & $0.41 ; 2.41$ & $0.75 ; 5.11$ & $0.45 ; 1.98$ \\
\hline \multirow[t]{2}{*}{ Serum pentosidine $(\mathrm{nmol} / \mathrm{l})^{c}$} & $73.6^{\mathrm{a}}$ & 27.1 & $71.5^{\mathrm{a}}$ & 30.3 \\
\hline & $48.4 ; 417.4$ & $15.46 ; 80.3$ & $31.4 ; 154.6$ & $16.2 ; 108.0$ \\
\hline
\end{tabular}

${ }^{\mathrm{a}}: p<0.001 ;^{\mathrm{b}}: p<0.005 ;^{\mathrm{c}}:$ Median a $95 \% \mathrm{Cl}$ 
were weighed and dried to a constant weight using a SpeedVac vacuum evaporator. The specimens of dry bone $(10 \mathrm{mg})$ were then hydrolyzed in $2 \mathrm{ml}$ of $6 \mathrm{M}$ hydrochloric acid at $105{ }^{\circ} \mathrm{C}$ for $16 \mathrm{~h}$. Collected blood serum samples were stored at $-20{ }^{\circ} \mathrm{C}$ until analysis.

\section{Pentosidine assay}

Pentosidine was determined by reversed phase HPLC combined with sensitive fluorescent detection, as published previously [21]. Briefly, serum samples were mixed with equal amounts of $35 \% \mathrm{HCl}$ and hydrolyzed for $16 \mathrm{~h}$ at $105{ }^{\circ} \mathrm{C}$. The bone samples were treated as above. In the next step, the hydrolysate was purified and pre-concentrated using solid phase extraction; the hydrolysate was mixed with a suspension of cellulose and combined with a solution containing n-butyl alcohol and acetic acid. This mixture was then applied to a purification column filled with cellulose. The PEN-containing fraction was then desorbed using $0.05 \mathrm{~mol} / \mathrm{l} \mathrm{HCl}$, and the extract was evaporated. The residue was reconstituted in a solution containing $0.02 \mathrm{~mol} / \mathrm{l}$ heptafluorobutyric acid, $0.01 \mathrm{~mol} / \mathrm{l}$ ammonium sulphate and acetonitrile (ergo mobile phase), and this was applied to a HPLC system (Shimadzu, LC-10ADvp, Kyoto, Japan) equipped with a C18 reversed phase column (Separon SGX C18, $150 \mathrm{x}$ $3 \mathrm{~mm}$ ). We monitored the emission signal at $385 \mathrm{~nm}$ upon excitation at $335 \mathrm{~nm}$. Synthetic PEN was used as a standard. The concentration of PEN was expressed in nmol/l.

The peak area reproducibility involving complete analytical procedure (including sample hydrolysis, drying, reconstitution, and HPLC analysis) in actual samples was $95.6 \%$. Reproducibility of the HPLC determination itself was $98.8 \%$. Based on our PEN measurements, limit of detection was $1.76 \mathrm{nmol} / \mathrm{l}$ and limit of quantitation $5.87 \mathrm{nmol} / \mathrm{l}$.

\section{Data analyses and statistical methods}

The sample size was estimated to enable detection of a difference in PEN between the two groups of patients. Results are expressed as percentages for categorical variables and as medians (95\% CI) or means (standard deviation, S.D.) for continuous variables. Between-group comparison of data was performed using the independent Student's $t$-test for continuous variables. A linear regression method analyzing the relationship between two variables was used; statistical significance of the correlation was determined by Pearson coefficients. Multiple linear regressions were used to estimate the differences in serum and bone PEN between fracture and osteoarthritis patients after adjusting for the effect of confounders such as age, sex, weight, serum creatinine, and diabetes. Values of $p<0.05$ were considered significant. Statistical analysis of data was performed using SigmaPlot 10 software (Systat Software Inc, Germany).

\section{Results}

In osteoarthritis cases, a growing trend of serum and bone PEN with age was found. In addition, a growing trend of serum PEN with age was found in individuals with fractures (Fig. 1). In fracture as well as in osteoarthritis patients, no significant correlation was found between serum creatinine and serum or bone PEN. No significant difference was found in PEN content in dry bone (using paired samples) from the femoral head and iliac crest. Serum 25-hydroxyvitamin D was measured in 46 fracture patients, concentrations $<25 \mathrm{nmol} / \mathrm{l}$ were found in $22 \%$ of them.

Serum and bone concentrations of PEN were significantly higher in subjects with hip fractures compared with osteoarthritis patients (Fig. 2). Significant differences in serum and bone PEN between fracture and osteoarthritis patients remained even after adjustment
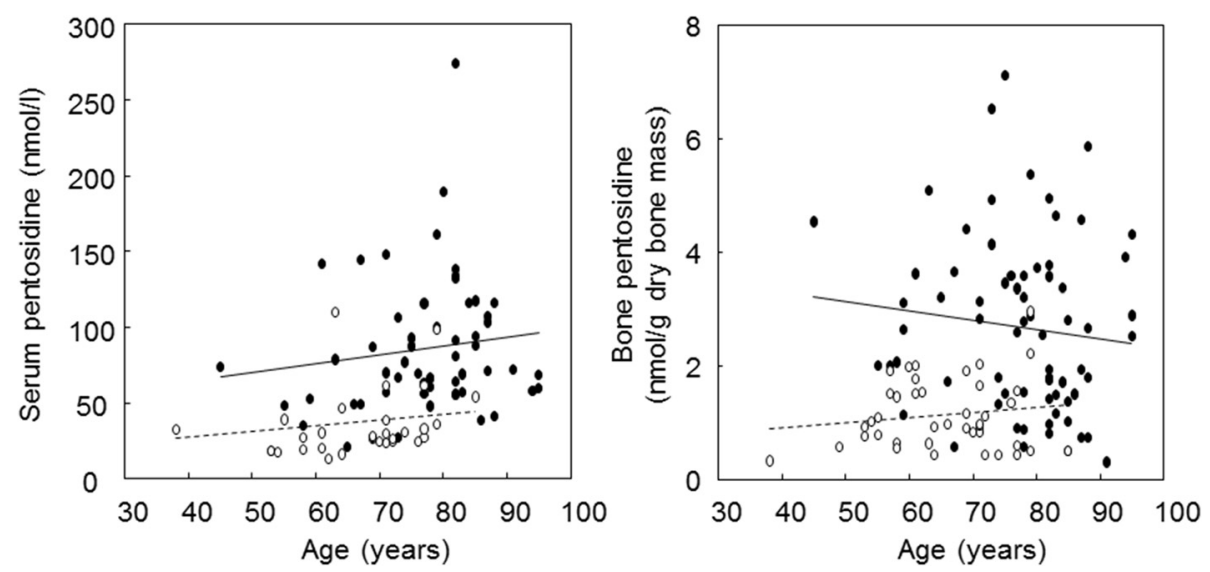

Fig. 1 Correlations between age and pentosidine levels in serum and bone in patients with hip fracture (full circles, serum, $r=0.13$, bone, $r=0.11$ ) and osteoarthritis patients (empty circles, serum, $r=0.14$, bone, $r=0.14$ ), n.s. for all 

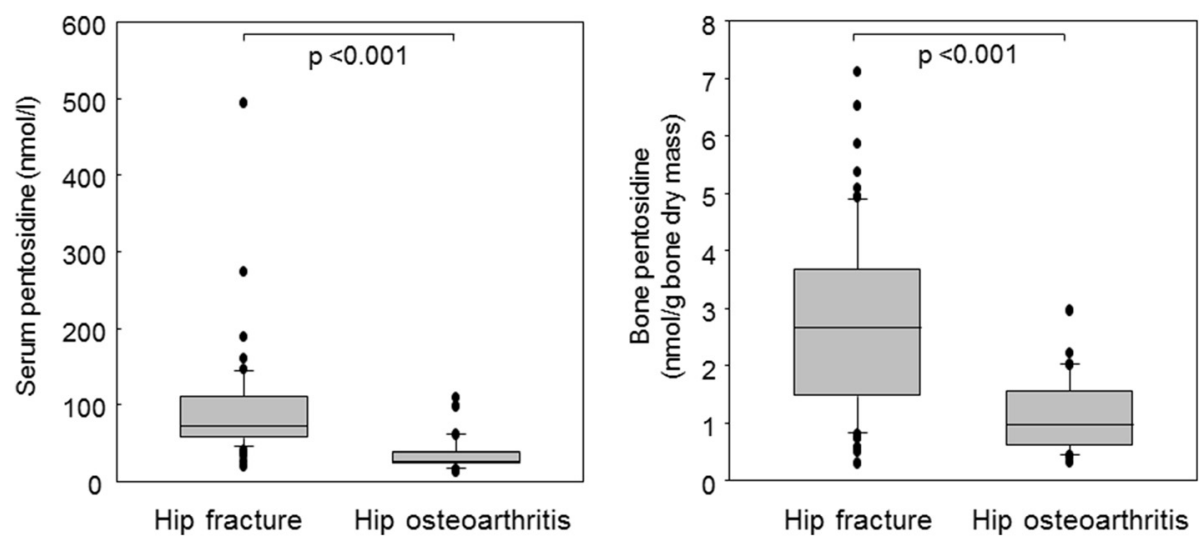

Fig. 2 Pentosidine content in serum and bone in patients with hip fracture and in hip osteoarthritis patients

for age, sex, weight, serum creatinine, and diabetes $(p<$ $0.001)$. A significant positive correlation was found between bone and serum PEN in fracture patients (Fig. 3). Receiver Operating Characteristics (ROC) curves indicated a comparable performance of serum and bone PEN in differentiation between fracture cases and osteoarthritis patients (Fig. 4).

\section{Discussion}

Previous studies have demonstrated that histomorphometric indices of trabecular bone micro-architecture were more favorable in patients undergoing arthroplasty for hip osteoarthritis than in patients with hip fracture, as well as in comparison with age-matched subjects [22-25]. In this study, bone PEN levels, considered surrogate markers of quality of organic bone matrix, were measured to assess another determinant of strength of bone, the bone tissue material properties. To our knowledge, this is the first study comparing serum and bone pentosidine levels in patients with low-impact hip fractures and in patients with advanced osteoarthritis. Our patients with hip fractures had higher

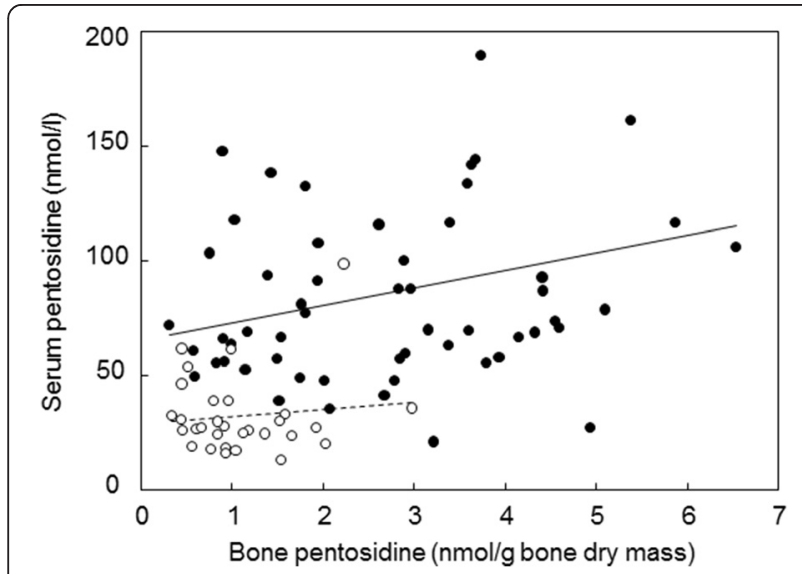

Fig. 3 Correlation between bone and serum pentosidine in osteoarthritis patients (empty circles, dotted line, $r=0.11$, n.s.) and in fractured cases (full circles and line, $r=0.27, p<0.05$ ) serum and bone PEN concentrations than those with advanced stages of hip osteoarthritis. Previously, PEN content in cortical and trabecular bone of patients with femoral neck fractures has been shown to be higher than in those of agematched controls $[18,26]$. However, the proof of the association between the risk of hip fractures and serum and bone PEN levels would require further studies, considering also bone microarchitecture, bone mineral density (BMD), clinical factors of risk of fracture and namely the general frailty of hip fracture patients should be considered [27, 28].

The association between bone PEN and fracture risk appears independent of BMD mainly in type-I diabetes [2931]. Also, using an Enzyme-Linked ImmunoSorbent Assay (ELISA), the association between serum PEN levels and changes in BMD in patients treated with bisphosphonate

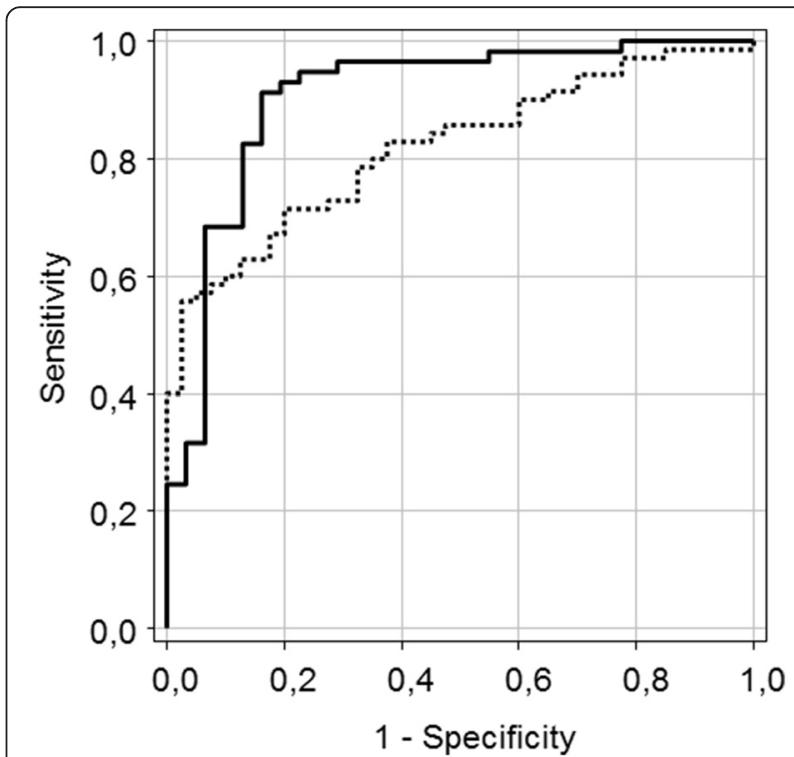

Fig. 4 Performance comparison of serum pentosidine, $A \cup C=0.906$ (full line) and bone pentosidine, $\mathrm{AUC}=0.845$ (dotted line), $p=0.274$. AUC: area under the curve 
was not confirmed [32]. However, in Japanese postmenopausal women, only a moderate BMD-independent association between urinary PEN and the incidence of vertebral and non-vertebral fractures was observed [28, 33, 34]. In the French OFELY study, there was a significant association between urinary PEN and the risk of all fractures in 396 healthy postmenopausal women, although, the odds ratio was not significant after adjustment for BMD [35].

This study has several limitations. First, the results in our patients must be interpreted within the context of differences in age and body weight, as well as the admitted risk factors for hip osteoarthritis, hip osteoporosis and fractures, although our results were adjusted for age, sex, weight, serum creatinine, and diabetes. The increasing trend in serum and bone PEN levels with age, which were observed in this study, was in accordance with that seen in other studies using HPLC [10], or ELISA [5, 36, 37]. Second, for ethical reasons, bone PEN levels in our patients were not compared with those in healthy age matched subjects from the general population. There was limited data on the differences between PEN levels in osteoarthritis and in healthy subjects, except for an observation that found no significant differences in plasma PEN levels in 17 patients with osteoarthritis compared with controls [19]. Previously, we reported that in patients with knee osteoarthritis, PEN serum concentrations were increased compared with healthy controls, and correlated with a cartilage destruction marker (cartilage oligomeric matrix protein) in synovial fluid [38]. However, knee osteoarthritis is mostly hypertrophic while in the majority of our patients, hip osteoarthritis was mostly of the atrophic type. Thus, although the interpretation of serum PEN levels in patients with knee and hip osteoarthritis and hip fracture should be done with caution, pentosidine crosslinking can nonetheless be considered as a measure of local bone age. Accordingly, serum PEN levels were higher in osteoporotic patients with long term aminobisphosphonate treatment than in BMD-matched controls [39]. Pentosidine formation is closely linked not only to glycation but also to oxidative processes [40, 41]. Accumulation of advanced non-enzymatic glycation end products in numerous body tissues during the course of aging, in diabetes, and in rheumatoid arthritis has been associated with oxidative stress [13]. It remains unknown whether the increase in AGE modified proteins in patients with chronic inflammation merely represents oxidative stress or they actively participate in bone and joint destruction [19]. Also, it is unclear to what extent serum PEN contributes to bone strength at fractured sites, since bone fractures are strain-controlled and PEN affects the organic matrix, which is better known as a contributor to post-yield deformation of bone rather than to its elastic properties [9]. Third, PEN has been shown to be also secreted from tissues other than bone and it is unclear what proportion of serum PEN levels actually reflects PEN content in bone. Compared with controls, PEN levels are significantly increased not only in aging, osteoporosis, diabetes mellitus, renal disorders, and rheumatoid arthritis [4, 5, 42-45], but have also been reported to correlate with the radiographic severity of lumbar spondylosis without vertebral fractures [46]. Increased serum PEN was an independent risk factor for loss of muscle mass in postmenopausal women with type 2 diabetes [47]. Finally, BMD measurements or histomorphometry of tetracycline labeled bone could not be performed in our patients with incident hip fractures, or those undergoing arthroplasty for hip osteoarthritis. Serum markers such as type 1 collagen cross-linked Ctelopeptide ( $\beta C T X)$ and procollagen type I aminoterminal propeptide (PINP) were not measured in our patients, since both markers are strongly affected by incident fractures. These markers reflect whole body rates of bone resorption and bone formation rather than the rates of remodeling at specific skeletal sites containing different ratios of trabecular to cortical component, each with its own metabolic rates [48].

\section{Conclusions}

Serum pentosidine can be considered a potential biomarker for identification of subjects with impaired quality of organic bone matrix. However, more studies are necessary to define the predictive value of serum PEN concerning fractures and the efficacy and safety of boneactive drugs in osteoporosis therapy.

\section{Abbreviations}

AGES, advanced glycation end-products; BMD, bone mineral density; ELISA, enzyme-linked immunosorbent assay; HPLC, high performance liquid chromatography; PEN, pentosidine; PINP, procollagen type I amino-terminal propeptide; ROC, Receiver Operating Characteristics; $\beta C T X$, type 1 collagen cross-linked C-telopeptide

\section{Acknowledgement}

This investigation was supported by the project for conceptual development of research organization 00023728 (Institute of Rheumatology, Prague, Czech Republic). The authors would like to thank Alena Stáňová for her excellent technical assistance in this project.

Funding

Ministry of Health, Czech Republic.

Availability of data and materials

Data and materials are available on request from JV.

Authors' contributions

$J V, M B$ and JS designed the study and drafted the manuscript. MB performed the biochemical analysis. JV selected the patients, and took written informed consent from patients and got the ethic committee. PD and KP participated in the coordination of the study and helped revise the manuscript. All authors read and approved the final manuscript. 


\section{Competing interests}

The authors declare that the research was conducted in the absence of any commercial or financial relationships that could be construed as a potential conflict of interest.

\section{Consent for publication}

Not applicable.

\section{Ethics approval and consent to participate}

Informed consent to biological materials for research was given at the time of surgery by the patients themselves. Consent forms were in accordance with procedures required by the ethics committee of the Institute of Postgradual Medical Education, Prague (MZ0 0002384101). All study methods and procedures were conducted in accordance with the Declaration of Helsinki

\section{Author details}

${ }^{1}$ Department of Orthopedics, Faculty of Medicine 1, Charles University Prague, and Bulovka Hospital, Prague, Czech Republic. ${ }^{2}$ Department of Composites and Carbon Materials, Institute of Rock Structure and Mechanics, Academy of Sciences of the Czech Republic, Prague, Czech Republic. ${ }^{3}$ Institute of Rheumatology, Prague, and Faculty of Medicine 1, Charles University Prague, Na Slupi 4, Prague CZ 12850, Czech Republic.

\section{Received: 12 April 2016 Accepted: 12 July 2016}

\section{Published online: 22 July 2016}

\section{References}

1. Leali PT, Muresu F, Melis A, Ruggiu A, Zachos A, Doria C. Skeletal fragility definition. Clin Cases Miner Bone Metab. 2011;8(2):11-3.

2. Garnero P. The contribution of collagen crosslinks to bone strength. BoneKey Rep. 2012;1:182.

3. Seeman E, Delmas PD. Bone quality - the material and structural basis of bone strength and fragility. N Engl J Med. 2006;354(21):2250-61.

4. Saito M, Kida Y, Kato S, Marumo K. Diabetes, collagen, and bone quality. Curr Osteoporos Rep. 2014:12(2):181-8.

5. Ikegami S, Uchiyama S, Nakamura Y, et al. Factors that characterize bone health with aging in healthy postmenopausal women. J Bone Miner Metab. 2015;33(4):440-7.

6. Allen MR, Gineyts E, Leeming DJ, Burr DB, Delmas PD. Bisphosphonates alter trabecular bone collagen cross-linking and isomerization in beagle dog vertebra. Osteoporos Int. 2008;19(3):329-37.

7. Bailey AJ, Paul RG, Knott L. Mechanisms of maturation and ageing of collagen. Mech Ageing Dev. 1998;106(1-2):1-56.

8. Vashishth D, Gibson GJ, Khoury JI, Schaffler MB, Kimura J, Fyhrie DP. Influence of nonenzymatic glycation on biomechanical properties of cortical bone. Bone. 2001:28(2):195-201.

9. Vashishth D. Advanced glycation end-products and bone fractures. IBMS BoneKEy. 2009;6(8):268-78.

10. Odetti P, Rossi S, Monacelli F, et al. Advanced glycation end products and bone loss during aging. Ann N Y Acad Sci. 2005;1043:710-7.

11. Wang $X$, Shen $X$, Li X, Agrawal CM. Age-related changes in the collagen network and toughness of bone. Bone. 2002;31(1):1-7.

12. Hernandez CJ, Tang SY, Baumbach BM, et al. Trabecular microfracture and the influence of pyridinium and non-enzymatic glycation-mediated collagen cross-links. Bone. 2005;37(6):825-32

13. Sell DR, Monnier VM. Structure elucidation of a senescence cross-link from human extracellular matrix. Implication of pentoses in the aging process. J Biol Chem. 1989;264(36):21597-602.

14. Saito M, Marumo K. Collagen cross-links as a determinant of bone quality: a possible explanation for bone fragility in aging, osteoporosis, and diabetes mellitus. Osteoporos Int. 2010;21(2):195-214.

15. Saito M, Marumo K, Fujii K, Ishioka N. Single-column high-performance liquid chromatographic-fluorescence detection of immature, mature, and senescent cross-links of collagen. Anal Biochem. 1997:253(1):26-32.

16. Garnero P, Borel O, Gineyts E, et al. Extracellular post-translational modifications of collagen are major determinants of biomechanical properties of fetal bovine cortical bone. Bone. 2006;38(3):300-9.

17. Viquet-Carrin S, Roux JP, Arlot ME, et al. Contribution of the advanced glycation end product pentosidine and of maturation of type I collagen to compressive biomechanical properties of human lumbar vertebrae. Bone. 2006:39(5):1073-9.

18. Saito M, Fujii K, Soshi S, Tanaka T. Reductions in degree of mineralization and enzymatic collagen cross-links and increases in glycation-induced pentosidine in the femoral neck cortex in cases of femoral neck fracture. Osteoporos Int. 2006;17(7):986-95.

19. Miyata T, Ishiguro N, Yasuda Y, et al. Increased pentosidine, an advanced glycation end product, in plasma and synovial fluid from patients with rheumatoid arthritis and its relation with inflammatory markers. Biochem Biophys Res Commun. 1998;244(1):45-9.

20. Conrozier T, Merle-Vincent F, Mathieu P, et al. Epidemiological, clinical, biological and radiological differences between atrophic and hypertrophic patterns of hip osteoarthritis: a case-control study. Clin Exp Rheumatol. 2004;22(4):403-8.

21. Spacek P, Adam M. HPLC method for pentosidine determination in urine, serum, and tissues as a marker of glycation and oxidation loading of the organism. J Liq Chromatogr Relat Technol. 2002;25:1807-20.

22. Blain $\mathrm{H}$, Chavassieux $\mathrm{P}$, Portero-Muzy N, et al. Cortical and trabecular bone distribution in the femoral neck in osteoporosis and osteoarthritis. Bone. 2008:43(5):862-8.

23. Resmini G, Migliaccio S, Dalle Carbonare L, et al. Differential characteristics of bone quality and bone turnover biochemical markers in patients with hip fragility fractures and hip osteoarthritis: results of a clinical pilot study. Aging Clin Exp Res. 2011;23(2):99-105.

24. Li B, Aspden RM. Composition and mechanical properties of cancellous bone from the femoral head of patients with osteoporosis or osteoarthritis. J Bone Miner Res. 1997;12(4):641-51.

25. Li B, Aspden RM. Material properties of bone from the femoral neck and calcar femorale of patients with osteoporosis or osteoarthritis. Osteoporos Int. 1997;7(5):450-6.

26. Saito M, Fujii K, Marumo K. Degree of mineralization-related collagen crosslinking in the femoral neck cancellous bone in cases of hip fracture and controls. Calcif Tissue Int. 2006;79(3):160-8.

27. McCloskey E, Johansson H, Oden A, Kanis JA. Fracture risk assessment. Clin Biochem. 2012:45(12):887-93.

28. Tanaka S, Kuroda T, Saito M, Shiraki M. Urinary pentosidine improves risk classification using fracture risk assessment tools for postmenopausal women. J Bone Miner Res. 2011;26(11):2778-84.

29. Schwartz AV Garnero P, Hillier TA, et al. Pentosidine and increased fracture risk in older adults with type 2 diabetes. J Clin Endocrinol Metab. 2009;94(7): 2380-6.

30. Yamamoto M, Yamaguchi T, Yamauchi M, Yano S, Sugimoto T. Serum pentosidine levels are positively associated with the presence of vertebral fractures in postmenopausal women with type 2 diabetes. J Clin Endocrinol Metab. 2008;93(3):1013-9.

31. Neumann T, Lodes $S$, Kastner $B$, et al. High serum pentosidine but not esRAGE is associated with prevalent fractures in type 1 diabetes independent of bone mineral density and glycaemic control. Osteoporos Int. 2014;25(5):1527-33.

32. Hashidate $H$, Kamimura M, Ikegami $S$, et al. Serum pentosidine levels after 3 years of bisphosphonate treatment in post-menopausal osteoporotic women. Endocr Res. 2015:40(3):172-6.

33. Shiraki M, Kuroda T, Tanaka S, Saito M, Fukunaga M, Nakamura T. Nonenzymatic collagen cross-links induced by glycoxidation (pentosidine) predicts vertebral fractures. J Bone Miner Metab. 2008;26(1):93-100.

34. Shiraki M, Kuroda T, Shiraki Y, Tanaka S, Higuchi T, Saito M. Urinary pentosidine and plasma homocysteine levels at baseline predict future fractures in osteoporosis patients under bisphosphonate treatment. J Bone Miner Metab. 2011;29(1):62-70.

35. Gineyts E, Munoz F, Bertholon C, Sornay-Rendu E, Chapurlat R. Urinary levels of pentosidine and the risk of fracture in postmenopausal women: the OFELY study. Osteoporos Int. 2010;21(2):243-50.

36. Yoshihara K, Nakamura K, Kanai M, et al. Determination of urinary and serum pentosidine and its application to elder patients. Biol Pharm Bull. 1998:21(10):1005-8.

37. Takahashi M, Oikawa M, Nagano A. Effect of age and menopause on serum concentrations of pentosidine, an advanced glycation end product. J Gerontol A Biol Sci Med Sci. 2000;55(3):M137-40.

38. Senolt L, Braun M, Olejarova M, Forejtova S, Gatterova J, Pavelka K. Increased pentosidine, an advanced glycation end product, in serum and synovial fluid from patients with knee osteoarthritis and its relation with cartilage oligomeric matrix protein. Ann Rheum Dis. 2005;64(6):886-90. 
39. Uchiyama S, Ikegami S, Kamimura M, et al. The skeletal muscle cross sectional area in long-term bisphosphonate users is smaller than that of bone mineral density-matched controls with increased serum pentosidine concentrations. Bone. 2015;75:84-7.

40. Dyer DG, Blackledge JA, Thorpe SR, Baynes JW. Formation of pentosidine during nonenzymatic browning of proteins by glucose. Identification of glucose and other carbohydrates as possible precursors of pentosidine in vivo. J Biol Chem. 1991;266(18):11654-60.

41. Wolff SP, Dean RT. Glucose autoxidation and protein modification. The potential role of 'autoxidative glycosylation' in diabetes. Biochem J. 1987; 245(1):243-50

42. Miyata T, lida Y, Horie K, Cai Z, Sugiyama S, Maeda K. Pathophysiology of advanced glycation end-products in renal failure. Nephrol Dial Transplant. 1996;11 Suppl 5:27-30.

43. Chen JR, Takahashi M, Suzuki M, Kushida K, Miyamoto S, Inoue T. Pentosidine in synovial fluid in osteoarthritis and rheumatoid arthritis: relationship with disease activity in rheumatoid arthritis. J Rheumatol. 1998;25(12):2440-4.

44. Rodriguez-Garcia J, Requena JR, Rodriguez-Segade S. Increased concentrations of serum pentosidine in rheumatoid arthritis. Clin Chem. 1998;44(2):250-5.

45. Hein GE, Kohler M, Oelzner P, Stein G, Franke S. The advanced glycation end product pentosidine correlates to IL- 6 and other relevant inflammatory markers in rheumatoid arthritis. Rheumatol Int. 2005;26(2):137-41.

46. Chiba D, Wada K, Tanaka T, et al. Serum pentosidine concentration is associated with radiographic severity of lumbar spondylosis in a general Japanese population. J Bone Miner Metab. 2015.

47. Tanaka K, Kanazawa I, Sugimoto T. Elevated Serum Pentosidine and Decreased Serum IGF-I Levels are Associated with Loss of Muscle Mass in Postmenopausal Women with Type 2 Diabetes Mellitus. Exp Clin Endocrinol Diabetes. 2015;124(3):163-6.

48. Delmas PD, Eastell R, Garnero P, Seibel MJ, Stepan J. The use of biochemical markers of bone turnover in osteoporosis. Committee of Scientific Advisors of the International Osteoporosis Foundation. Osteoporos Int. 2000;11(6):S2-17.

\section{Submit your next manuscript to BioMed Central and we will help you at every step:}

- We accept pre-submission inquiries

- Our selector tool helps you to find the most relevant journal

- We provide round the clock customer support

- Convenient online submission

- Thorough peer review

- Inclusion in PubMed and all major indexing services

- Maximum visibility for your research

Submit your manuscript at www.biomedcentral.com/submit

) Biomed Central 